\title{
Performance of Ridge Estimators Based on Weighted Geometric Mean and Harmonic Mean
}

\author{
S. S. Bhat, R. Vidya* \\ Department of Statistics, Yuvaraja's College, University of Mysore, Mysuru, Karnataka, India \\ Received 9 March 2019, accepted in final revised form 12 September 2019
}

\begin{abstract}
Ordinary least squares estimator (OLS) becomes unstable if there is a linear dependence between any two predictors. When such situation arises ridge estimator will yield more stable estimates to the regression coefficients than OLS estimator. Here we suggest two modified ridge estimators based on weights, where weights being the first two largest eigen values. We compare their MSE with some of the existing ridge estimators which are defined in the literature. Performance of the suggested estimators is evaluated empirically for a wide range of degree of multicollinearity. Simulation study indicates that the performance of the suggested estimators is slightly better and more stable with respect to degree of multicollinearity, sample size, and error variance.
\end{abstract}

Keywords: Multivariate linear regression (MLR); Multicollinearity; Ridge regression; Weights; MSE.

(C) 2020 JSR Publications. ISSN: 2070-0237 (Print); 2070-0245 (Online). All rights reserved.

doi: http://dx.doi.org/10.3329/jsr.v12i1.40525 J. Sci. Res. 12 (1), 1-13 (2020)

\section{Introduction}

Consider the standard form of multivariate linear regression (MLR) model defined by

$$
\boldsymbol{y}=X \boldsymbol{\beta}+\boldsymbol{u}
$$

Where $X$ is $(n \times p)$ data matrix, $\boldsymbol{y}$ is $(n \times 1)$ vector of response, $\boldsymbol{\beta}$ is $(p \times 1)$ vector of regression coefficients and $\boldsymbol{u}$ is $(n \times 1)$ vector of residuals which are i.i.d. with zero mean and variance $\sigma^{2}$. When $X$ has full rank, the ordinary least squares (OLS) method, gives the estimate for $\boldsymbol{\beta}$ as

$$
\hat{\boldsymbol{\beta}}_{O L S}=\left(X^{\prime} X\right)^{-1} X^{\prime} \boldsymbol{y}
$$

Due to near linear dependence between the predictors, $\left(X^{\prime} X\right)^{-1}$ may not exist always and therefore OLS estimator will yield unstable estimates to the regression coefficients. That is, in presence of multicollinearity, OLS estimator becomes biased and also has large variance. When moderate or severe degree of multicollinearity is present

\footnotetext{
${ }^{*}$ Corresponding author: drvidyaraju02@gmail.com
} 
var $-\operatorname{cov}\left(\hat{\boldsymbol{\beta}}_{\text {OLS }}\right)=\sigma^{2}\left(X^{\prime} X\right)^{-1}$ will be large. That is, regression parameters are sensitive to small changes in the response variable $\boldsymbol{y}$ or the matrix of predictors $X$ (Marquardt and Snee, [1]; Vinod and Ullah, [2]). Thus to overcome the drawbacks of OLS, there are number of techniques have been proposed in the literature, viz., Ridge regression, Principal component regression, Partial least squares regression, LASSO method of regression, Liu method of regression etc., for details, see Hoerl [3], Hoerl and Kennard [4], Helland [5], Vinod and Ullah [2], Mardikyan and Cetin [6], Tibshirani [7].

\section{The Canonical Model}

Here matrix $X$, is standardised such that $X^{\prime} X$, is in the form of a near correlation matrix. Further, let $Z=X W$ where $W$ is a matrix of order $(p \times p)$ so that its columns are normalized eigen vectors of $X^{\prime} X$, such that $Z^{\prime} Z=W^{\prime} X^{\prime} X W=D=\operatorname{diag}\left(\lambda_{1}, \lambda_{2}, \ldots, \lambda_{p}\right)$, where $\lambda_{j}{ }^{\prime} s$ are the $j^{\text {th }}$ eigen value of $X^{\prime} X$. The equation (1) becomes

$$
\boldsymbol{y}=Z \gamma+\boldsymbol{u},
$$

where $\boldsymbol{\gamma}=W^{\prime} \boldsymbol{\beta}$. The OLS estimator for $\gamma$ is then given by

$$
\hat{\gamma}_{O L S}=\left(Z^{\prime} Z\right)^{-1} Z^{\prime} \boldsymbol{y}=D^{-1} Z^{\prime} \boldsymbol{y} .
$$

Since $\boldsymbol{\gamma}=W^{\prime} \boldsymbol{\beta}$, implies $\hat{\boldsymbol{\beta}}=W \hat{\boldsymbol{\gamma}}$.

\section{Ridge Estimation}

Ridge regression is one of the most widely used techniques to cope with the problem of multicollinearity. It is an alternative technique to OLS. OLS estimator become unstable when there is a linear dependence between any two predictors. Linear dependency leads to multicollinearity and thereby inverse of the matrix $X^{\prime} X$, may not be possible. When such a situation arises, Hoerl and Kennard [4] have suggested that; add a constant $k(>0)$ to every $j^{\text {th }}$ element of the diagonal of the matrix $X^{\prime} X$, to overcome the problem of singularity. That is increasing the length of OLS estimator by a small amount $k(>0)$, will reduce the MSE of $\hat{\boldsymbol{\beta}}$, and such a modification in $X^{\prime} X$ made the ridge estimator more robust to the problem of singularity.

The ordinary ridge estimator for the regression coefficients is given by

$$
\hat{\gamma}_{R}=\left(Z^{\prime} Z+k I\right)^{-1} Z^{\prime} \boldsymbol{y}=A^{-1} Z^{\prime} \boldsymbol{y}
$$

Where $A=D+k I$ and $X=Z W^{\prime}$. Using equation (4), equation (5) reduce to

$$
\hat{\gamma}_{R}=\left(I-A^{-1} k\right) \hat{\gamma}_{O L S} .
$$

Hoerl and Kennard [4] showed that ridge estimator is biased and its bias-square is continuous and monotonically increasing function of $k$. Also for $0 \leq k \leq \frac{\sigma^{2}}{\hat{\gamma}_{\max }^{2}}$, the MSE 
$\left(\hat{\gamma}_{R}\right)$ is minimum, where $\hat{\gamma}_{\max }^{2}$ is the largest element of $\hat{\gamma}_{R}^{2}$ and $\sigma^{2}$ is replaced by its estimate $\hat{\sigma}^{2}=\frac{y^{\prime} \boldsymbol{y}-\hat{\gamma}_{O L S}^{\prime} Z^{\prime} \boldsymbol{y}}{n-p-1}$. Halawa and El Bassiouni [8] suggested that the estimate of $\sigma^{2}$ is $\hat{\sigma}^{2}=\frac{\boldsymbol{y}^{\prime} \boldsymbol{y}-\hat{\gamma}_{O L S}^{\prime} Z^{\prime} \boldsymbol{y}}{n-p}$. Above two estimators of $\sigma^{2}$, may yield negative estimates to the residual mean square, if $n<p$. Instead, herewith considered Hastie and Tibshirani's [9] methodology, which makes the use of 'hat matrix', $H$, in turn the degrees of freedom for the error is $n-\operatorname{tr}\left(2 H-H H^{\prime}\right)=n-p$.

\subsection{Some well-known ridge estimators}

In the literature, several authors have defined different methods of estimating the ridge parameter $k$, to overcome the problem of multicollinearity. Some of the well-known methods for choosing ridge parameter are due to references [2,10-17].

Some of the well-known methods of estimating the ridge parameter $k$ are

i) $k_{1}=\frac{p \hat{\sigma}^{2}}{\hat{\gamma}^{\prime} \hat{\gamma}}$

(Hoerl, Kennard, and Baldwin, [18])

where $p$, is the number of predictors, $\hat{\sigma}^{2}$ is the estimate of $\sigma^{2}$ and $\hat{\gamma}$ is the estimate of $\gamma$.

ii) $k_{2}=\frac{p \hat{\sigma}^{2}}{\sum_{j=1}^{p} \lambda_{j} \hat{\gamma}_{j}^{2}} \quad$ (Lawless and Wang, [10])

where $\lambda_{j}$ is the eigen $j^{\text {th }}$ value of $X^{\prime} X$.

iii) $k_{3}=\frac{p \hat{\sigma}^{2}}{\sum_{j=1}^{p}\left\{\hat{\gamma}_{j}^{2} /\left[1+\left(1+\lambda_{j}\left[\hat{\gamma}_{j}^{2} / \hat{\sigma}^{2}\right]^{1 / 2}\right)\right]\right.} \quad$ (Nomura, [19])

where $\lambda_{j}$ is the eigen $j^{\text {th }}$ value of $X^{\prime} X$.

iv) $k_{4}=\frac{\lambda_{\max } \hat{\sigma}^{2}}{(n-p-1) \hat{\sigma}^{2}+\lambda_{\max } \hat{\gamma}_{\max }^{2}}, \quad$ (Khalaf and Shukur, [12])

where $\lambda_{\max }$ the largest Eigen is value of $X^{\prime} X$, and $\hat{\gamma}_{\max }^{2}$ is the largest element of $\hat{\gamma}_{R}^{2}$

v) $k_{5}=\operatorname{Max}\left(0, \frac{p \hat{\sigma}^{2}}{\hat{\gamma}^{\prime} \hat{\gamma}}-\frac{1}{n\left(V I F_{j}\right)_{\max }}\right)$, (Dorugade and Kashid, [15])

where $V I F_{j}=\frac{1}{\left(1-R_{j}^{2}\right)}, j=1,2, \ldots, p$; is the variance inflation factor of the $j^{\text {th }}$ regressor. 
vi) $k_{6}=\frac{2 p}{\lambda_{\max }} \sum_{j=1}^{p} \frac{\hat{\sigma}^{2}}{\hat{\gamma}_{j}^{2}},($ Dorugade, $[16])$

where $\lambda_{\max }$ is the largest eigen value of $X^{\prime} X$.

vii) $k_{7}=\frac{p \hat{\sigma}^{2}}{\hat{\gamma}^{\prime} \hat{\gamma}}+\frac{1}{n \lambda_{\max }}=k_{1}+\frac{1}{n \lambda_{\max }},($ Satish and Vidya, [20])

where $\lambda_{\max }$ is the largest eigen value of $X^{\prime} X$.

viii) $k_{8}=\frac{p \hat{\sigma}^{2}}{\hat{\gamma}^{\prime} \hat{\gamma}}+\frac{1}{\lambda_{\max } \hat{\gamma}^{\prime} \hat{\gamma}}=k_{1}+\frac{1}{\lambda_{\max } \hat{\gamma}^{\prime} \hat{\gamma}}, \quad$ (Satish and Vidya, [21])

where $\lambda_{\max }$ is the largest eigen value of $X^{\prime} X$.

ix) $k_{9}=\frac{p \hat{\sigma}^{2}}{\hat{\gamma}^{\prime} \hat{\gamma}}+\frac{1}{2\left(\sqrt{\lambda_{\max } / \lambda_{\min }}\right)^{2}}=k_{1}+\frac{1}{2 m^{2}}$, (Satish and Vidya, [21])

where $m=\sqrt{\lambda_{\max } / \lambda_{\min }}$ is the condition number [2,22]. Higher the value of $m$, higher is the degree of multicollinearity. If $(30<m<100)$ means a moderate to strong multicollinearity, and if $m>100$ suggests severe multicollinearity [23].

x) $k_{10}=G M\left(k_{H K B}, k_{D K}\right)=\sqrt{k_{1} \times k_{5}}, \quad$ (Satish and Vidya, [24])

xi) $k_{11}=H M\left(k_{1}, k_{5}\right)=2 k_{1} \times k_{5} /\left(k_{1}+k_{5}\right), \quad($ Satish and Vidya, [24])

where, $k_{1}$ is the estimator due to Hoerl et al. [18] and $k_{5}$ is the estimator due to Dorugade and Kashid, [15].

xii) $k_{12}=W A M\left(k_{1}, k_{5}\right)=\left(\frac{w_{2} k_{1}+w_{1} k_{5}}{w_{1}+w_{2}}\right), \quad($ Satish, [25])

where, weight $w_{1}=\lambda_{1}$, being the largest eigen value, and $w_{2}=\lambda_{2}$, is the second largest eigen value of $X^{\prime} X$ matrix. It is observed that the estimators defined in equations (7) to (12) are verified under very high degree $(\rho \geq 0.9)$ of multicollinearity between the predictors whereas, the estimators due to Satish and Vidya [20,21,24] are investigated under various degree of multicollinearity viz., low, moderate and high degree of multicollinearity. Also, Satish and Vidya [20,24], have considered different error distributions viz., normal and non-normal $\left(t_{(5)}\right.$ - distribution with 5 d.f.) for the error term.

Further, the estimator due to Hoerl et al. [18] seems to be over shrunken the estimator towards zero and it does not work well when number of predictors is more than the sample size whereas the estimator due to Dorugade and Kashid [15] performs better when number of predictors is more than the sample size, and also when predictors are highly collinear, i.e., later is more stable than the earlier. Moreover, Askin and Montgomery [26], proposed ridge regression based on M-estimates, where the M-estimates were computed using weighted least squares procedure. Holland [27] suggests that weights being used are non-negative, may not be equal, and their sum need not be equal to unity. Holland [27] 
also used the weighted least squares procedure to estimate $\boldsymbol{\beta}$, where $\boldsymbol{\beta}$ 's are obtained by subtracting the means of $\boldsymbol{y}^{\prime}$ s and jth column of $X_{j}$, respectively. Motivated by these features of the above estimators, we would like to suggest a new method of assigning weights to ordinary ridge estimators.

\section{Proposed Estimators}

Here we suggest two modified ridge estimators namely, weighted geometric mean ridge estimator; and secondly, a weighted harmonic mean ridge estimator, where weights being the first two largest eigen values $\lambda_{1}$ and $\lambda_{2}\left(\lambda_{1}>\lambda_{2}\right)$. These two estimators are obtained by taking weighted geometric mean and weighted harmonic mean of the estimators due to Dorugade and Kashid [15], and Hoerl et al. [18] respectively. We have assigned higher weight ( $w_{1}=\lambda_{1}$, largest eigen value) to estimator, which is due to Dorugade and Kashid [15], and lower weight ( $w_{2}=\lambda_{2}$, the second largest eigen value) to estimator, which is due to Hoerl et al. [18]. The weights are assigned in such a way that prior is more stable than the later, for $n<p$; and the suggested estimators is defined by

$$
\begin{aligned}
& \text { xiii) } k_{13}=W G M\left(k_{1}, k_{5}\right)=\exp \left(\frac{w_{2} \ln \left(k_{1}\right)+w_{1} \ln \left(k_{5}\right)}{w_{1}+w_{2}}\right), \\
& \text { xiv) } k_{14}=W H M\left(k_{1}, k_{5}\right)=\frac{w_{1}+w_{2}}{\left(w_{2} / k_{1}\right)+\left(w_{1} / k_{5}\right)}=\frac{k_{1} k_{5} \sum_{i=1}^{2} w_{i}}{w_{2} k_{5}+w_{1} k_{1}},
\end{aligned}
$$

Modifications made the suggested estimators more robust to the problem of multicollinearity, since the two largest eigen values $\lambda_{1}$ and $\lambda_{2}$, carry most of the information's of the data matrix $X$ and further, it is observed that an input of higher weights to more stable estimator will have more influence on the MSE, that is, MSE of the suggested estimators gets reduced to a certain extent, and thereby suggested estimators become more stable and comparable to some of the existing estimators, which are considered under this study.

LASSO [7] technique of regression is the one which shrinks some regression coefficients in absolute terms and sets others to zero. That is "LASSO minimizes the residual sum of squares subject to the sum of the absolute value of the coefficients being less than a constant", [17]. It is also used as subset selection operator and hence "tries to retain the good features of both subset selection and ridge regression" [7]. This article pertains to weighted ridge regression and does not through light on the subset selection.

\section{Application of Real Data}

Here we consider a part of the real data related to wages and other characteristics of workers [28]. We have computed VIF to know the degree of multicollinearity between the predictors; and then computed average mean square error (AMSE) ratio of OLSE over 
different ridge estimators which are considered in this article. The simulation results are given in Tables 1 and 2 .

Table 1. VIF values of different predictors.

\begin{tabular}{llllllll}
\hline Predictors & $\mathrm{X}_{1}$ & $\mathrm{X}_{2}$ & $\mathrm{X}_{3}$ & $\mathrm{X}_{4}$ & $X_{5}$ & $X_{6}$ & $X_{7}$ \\
\hline VIF & 1.2982 & 1.1987 & 1.1209 & 231.1956 & 5184.0939 & 4645.6650 & 1.0916 \\
\hline
\end{tabular}

VIF values indicated that there exists a moderate to very high degree of multicollinearity between the predictors.

Table 2. AMSE of OLSE over other ridge estimators.

\begin{tabular}{|c|c|c|c|c|c|c|c|c|c|c|c|c|c|c|}
\hline Estimators & $k_{1}$ & $k_{2}$ & $k_{3}$ & $k_{4}$ & $k_{5}$ & $k_{6}$ & $k_{7}$ & $k_{8}$ & $k_{9}$ & $k_{10}$ & $k_{11}$ & $k_{12}$ & $k_{13}$ & $k_{14}$ \\
\hline AMSE & $\underset{\substack{+0}}{\stackrel{+}{+}}$ & $\begin{array}{l}\stackrel{0}{n} \\
\text { กิ } \\
\hat{0}\end{array}$ & $\begin{array}{l}\infty \\
\text { त̂ } \\
\infty \\
0 \\
0\end{array}$ & $\begin{array}{l}\hat{\infty} \\
\infty \\
\infty \\
0\end{array}$ & $\begin{array}{l}n \\
\infty \\
\infty \\
\infty \\
0 \\
0\end{array}$ & $\begin{array}{l}\infty \\
\stackrel{N}{N} \\
\infty \\
0 \\
0\end{array}$ & $\underset{\substack{J \\
0}}{\stackrel{J}{f}}$ & 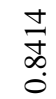 & $\begin{array}{l}\underset{+}{+} \\
\infty \\
0\end{array}$ & $\begin{array}{l}\underset{+}{+} \\
\stackrel{\infty}{0} \\
\dot{0}\end{array}$ & $\begin{array}{l}\underset{+}{+} \\
\stackrel{\infty}{0}\end{array}$ & $\begin{array}{l}\text { ळे } \\
\infty \\
\infty \\
0\end{array}$ & $\begin{array}{l}n \\
\infty \\
\infty \\
\infty \\
0 \\
0\end{array}$ & $\begin{array}{l}n \\
\infty \\
\infty \\
\infty \\
0\end{array}$ \\
\hline
\end{tabular}

Above results indicate that the suggested estimators $k_{13}$, and $k_{14}$, perform better than all the other estimators except, the estimator due to Dorugade and Kashid [15], i.e., $k_{5}$, but the two suggested estimators coincide with [15], in terms of MSE, and thereby their performances are comparable and satisfactory.

\section{Simulation Study}

Simulation study was conducted for various values of $n$, the sample size; $p$ the number of predictors, residual variance $\sigma^{2}$, and $\rho$, the degree of correlation, in the presence of low, moderate and a high degree of multicollinearity. The results were obtained by generating a random data matrix $X$ of size $(n \times p)$ using the relation:

$$
x_{i j}=\left(1-\rho^{2}\right)^{1 / 2} \xi_{i j}+\rho \xi_{i p}, i=1,2, \ldots, n ; j=1,2, \ldots, p ;
$$

here $\xi_{i j}$ 's are independent standard normal pseudo-random numbers, $\rho$ is fixed such that $\rho^{2}$ is the degree of correlation between any two predictors. These variables are standardized such that $X^{\prime} X$ takes up correlation matrix form, and to generate $y$ we have assumed vector $\boldsymbol{\beta}$ as $\beta=[0.03,0.5,0.03,0.91,0.59,0.74,0.3,0.95,0.83,0.9,0.5,0.4,0.3,0.5$, $0.3,0.9]^{\prime}$. The performance of the suggested estimators was evaluated with $n=25,50$, and $1000 ; p=15$, the variance of the residual term $\sigma^{2}: 5,25,100$, and 1000; and the degree of correlation $\rho=0.3,0.5,0.7,0.9,0.99$ and 0.9999 . The experiment was replicated 1000 times each and the average of mean square error (AMSE) was computed using the relation,

$$
\operatorname{AMSE}\left(\hat{\boldsymbol{\beta}}^{*}\right)=\frac{1}{1000} \sum_{j=1}^{1000}\left(\hat{\boldsymbol{\beta}}_{(j)}^{*}-\boldsymbol{\beta}\right)^{\prime}\left(\hat{\boldsymbol{\beta}}_{(j)}^{*}-\boldsymbol{\beta}\right)
$$


where, $\hat{\boldsymbol{\beta}}^{*}$ is any estimator that was used in this study. Ridge estimates were computed by considering the different estimators of the ridge parameter $k$, defined in equations (7) to (15). The results of the simulation are presented in Table 3. Here, the estimators leading to the maximum ratio of AMSE of OLS over AMSE of other ridge estimators were considered to be the best in terms of MSE.

Table 3. AMSE ratio of OLSE over different Ridge estimator's when error $(u) \sim \mathrm{N}\left(0, \sigma^{2} I\right)$.

\begin{tabular}{lllllllc}
\hline$n=25$ & AMSE & $\rho=0.3$ & $\rho=0.5$ & $\rho=0.7$ & $\rho=0.9$ & $\rho=0.99$ & $\rho=0.9999$ \\
\hline$\sigma^{2}=5$ & $k_{1}$ & 1.3847 & 1.5546 & 2.0207 & 3.0783 & 4.3582 & 4.4458 \\
& $k_{2}$ & 0.8151 & 0.9654 & 1.2828 & 2.4334 & 9.2939 & 8.4226 \\
& $k_{3}$ & 2.6436 & 2.8054 & 3.6249 & 6.1488 & 9.5922 & 8.0385 \\
& $k_{4}$ & 0.9547 & 1.0856 & 1.3709 & 1.7858 & 2.0074 & 2.0952 \\
& $k_{5}$ & 0.6536 & 0.7119 & 0.7950 & 0.9087 & 0.9972 & 1.0039 \\
& $k_{6}$ & 1.7419 & 2.1588 & 2.4789 & 3.4314 & 13.5524 & 9.3012 \\
& $k_{7}$ & 1.3848 & 1.5546 & 2.0207 & 3.0786 & 4.3631 & 4.9155 \\
& $k_{8}$ & 1.3848 & 1.5546 & 2.0207 & 3.0783 & 4.3583 & 4.4458 \\
& $k_{9}$ & 1.3862 & 1.5556 & 2.0215 & 3.0794 & 4.3600 & 4.4476 \\
& $k_{10}$ & 0.6536 & 0.7119 & 0.7950 & 0.9087 & 0.9972 & 1.0039 \\
& $k_{11}$ & 0.6536 & 0.7119 & 0.7950 & 0.9087 & 0.9972 & 1.0039 \\
& $k_{12}$ & 1.0641 & 1.0777 & 1.0861 & 1.1813 & 1.2852 & 1.2936 \\
& $k_{13}$ & 0.6536 & 0.7119 & 0.7950 & 0.9087 & 0.9972 & 1.0039 \\
& $k_{14}$ & 0.6536 & 0.7119 & 0.7950 & 0.9087 & 0.9972 & 1.0039 \\
\hline$\sigma^{2}=25$ & $k_{1}$ & 3.8362 & 4.0195 & 4.1562 & 4.5012 & 4.5178 & 4.5289 \\
& $k_{2}$ & 1.5163 & 1.6370 & 1.9547 & 3.4116 & 23.6202 & 35.0617 \\
& $k_{3}$ & 15.5810 & 15.7196 & 15.0989 & 13.8062 & 10.2328 & 8.2485 \\
& $k_{4}$ & 1.7682 & 1.8619 & 2.0544 & 2.1102 & 2.1038 & 2.1636 \\
& $k_{5}$ & 0.9827 & 0.9865 & 0.9920 & 1.0002 & 1.0036 & 1.0037 \\
& $k_{6}$ & 9.8250 & 7.8409 & 6.4622 & 8.1541 & 58.6202 & 64.2824 \\
& $k_{7}$ & 3.8366 & 4.0198 & 4.1564 & 4.5018 & 4.5229 & 5.0202 \\
& $k_{8}$ & 3.8362 & 4.0195 & 4.1562 & 4.5012 & 4.5178 & 4.5289 \\
& $k_{9}$ & 3.8441 & 4.0245 & 4.1593 & 4.5033 & 4.5197 & 4.5308 \\
& $k_{10}$ & 0.9827 & 0.9865 & 0.9920 & 1.0002 & 1.0036 & 1.0037 \\
& $k_{11}$ & 0.9827 & 0.9865 & 0.9920 & 1.0002 & 1.0036 & 1.0037 \\
& $k_{12}$ & 2.3191 & 1.9745 & 1.5516 & 1.3452 & 1.2967 & 1.2959 \\
& $k_{13}$ & 0.9827 & 0.9865 & 0.9920 & 1.0002 & 1.0036 & 1.0037 \\
& $k_{14}$ & 0.9827 & 0.9865 & 0.9920 & 1.0002 & 1.0036 & 1.0037 \\
\hline & & & & & & & \\
& & & & & \\
& & & &
\end{tabular}

Table 3. Continued... 


\begin{tabular}{|c|c|c|c|c|c|c|c|}
\hline \multirow[t]{14}{*}{$\sigma^{2}=100$} & $k_{1}$ & 4.1835 & 4.1850 & 4.2574 & 4.2754 & 4.4948 & 4.3794 \\
\hline & $k_{2}$ & 1.5571 & 1.6521 & 1.9876 & 3.3089 & 24.2098 & 36.4688 \\
\hline & $k_{3}$ & 19.4858 & 18.6403 & 17.0535 & 13.4637 & 10.1515 & 7.8843 \\
\hline & $k_{4}$ & 1.8143 & 1.9598 & 1.9854 & 2.0473 & 2.1504 & 2.0742 \\
\hline & $k_{5}$ & 1.0018 & 1.0016 & 1.0035 & 1.0039 & 1.0039 & 1.0039 \\
\hline & $k_{6}$ & 10.9368 & 8.4201 & 6.6092 & 8.6282 & 66.6886 & 69.2413 \\
\hline & $k_{7}$ & 4.1839 & 4.1853 & 4.2577 & 4.2762 & 4.5003 & 5.0290 \\
\hline & $k_{8}$ & 4.1835 & 4.1850 & 4.2574 & 4.2754 & 4.4948 & 4.3794 \\
\hline & $k_{9}$ & 4.1917 & 4.1902 & 4.2609 & 4.2778 & 4.4967 & 4.3817 \\
\hline & $k_{10}$ & 1.0018 & 1.0016 & 1.0035 & 1.0039 & 1.0039 & 1.0039 \\
\hline & $k_{11}$ & 1.0018 & 1.0016 & 1.0035 & 1.0039 & 1.0039 & 1.0039 \\
\hline & $k_{12}$ & 2.4601 & 2.0291 & 1.5672 & 1.3311 & 1.3014 & 1.2877 \\
\hline & $k_{13}$ & 1.0018 & 1.0016 & 1.0035 & 1.0039 & 1.0039 & 1.0039 \\
\hline & $k_{14}$ & 1.0018 & 1.0016 & 1.0035 & 1.0039 & 1.0039 & 1.0039 \\
\hline \multirow[t]{14}{*}{$\sigma^{2}=1000$} & $k_{1}$ & 4.2053 & 4.2322 & 4.4203 & 4.4399 & 4.3937 & 4.4939 \\
\hline & $k_{2}$ & 1.5556 & 1.7040 & 2.0094 & 3.3465 & 22.7244 & 32.1036 \\
\hline & $k_{3}$ & 20.0472 & 18.5617 & 17.4034 & 14.0752 & 9.8729 & 8.2017 \\
\hline & $k_{4}$ & 1.8110 & 1.9661 & 2.0098 & 2.0775 & 2.0975 & 2.1428 \\
\hline & $k_{5}$ & 1.0023 & 1.0032 & 1.0035 & 1.0039 & 1.0037 & 1.0040 \\
\hline & $k_{6}$ & 11.9794 & 8.4695 & 6.6124 & 9.2420 & 57.5703 & 58.1728 \\
\hline & $k_{7}$ & 4.2057 & 4.2326 & 4.4206 & 4.4404 & 4.3998 & 4.9828 \\
\hline & $k_{8}$ & 4.2053 & 4.2322 & 4.4203 & 4.4399 & 4.3937 & 4.4939 \\
\hline & $k_{9}$ & 4.2132 & 4.2376 & 4.4235 & 4.4421 & 4.3956 & 4.4957 \\
\hline & $k_{10}$ & 1.0023 & 1.0032 & 1.0035 & 1.0039 & 1.0037 & 1.0040 \\
\hline & $k_{11}$ & 1.0023 & 1.0032 & 1.0035 & 1.0039 & 1.0037 & 1.0040 \\
\hline & $k_{12}$ & 2.4572 & 2.0240 & 1.5939 & 1.3444 & 1.2999 & 1.2920 \\
\hline & $k_{13}$ & 1.0023 & 1.0032 & 1.0035 & 1.0039 & 1.0037 & 1.0040 \\
\hline & $k_{14}$ & 1.0023 & 1.0032 & 1.0035 & 1.0039 & 1.0037 & 1.0040 \\
\hline \multirow[t]{14}{*}{$\sigma^{2}=5$} & $k_{1}$ & 0.4921 & 0.5460 & 0.7318 & 1.5173 & 3.5282 & 4.1053 \\
\hline & $k_{2}$ & 0.3547 & 0.4027 & 0.5111 & 0.9051 & 4.4414 & 21.3857 \\
\hline & $k_{3}$ & 0.9480 & 0.9960 & 1.2204 & 2.7386 & 9.7035 & 8.7063 \\
\hline & $k_{4}$ & 0.3731 & 0.4314 & 0.5718 & 1.0255 & 1.7613 & 1.8971 \\
\hline & $k_{5}$ & 0.3463 & 0.3894 & 0.4806 & 0.7251 & 0.9704 & 1.0037 \\
\hline & $k_{6}$ & 0.5295 & 0.5773 & 0.6542 & 1.0676 & 4.6746 & 15.1524 \\
\hline & $k_{7}$ & 0.4921 & 0.5460 & 0.7318 & 1.5173 & 3.5284 & 4.1246 \\
\hline & $k_{8}$ & 0.4921 & 0.5460 & 0.7318 & 1.5173 & 3.5282 & 4.1053 \\
\hline & $k_{9}$ & 0.4925 & 0.5462 & 0.7320 & 1.5176 & 3.5291 & 4.1064 \\
\hline & $k_{10}$ & 0.3463 & 0.3894 & 0.4806 & 0.7251 & 0.9704 & 1.0037 \\
\hline & $k_{11}$ & 0.3463 & 0.3894 & 0.4806 & 0.7251 & 0.9704 & 1.0037 \\
\hline & $k_{12}$ & 0.4181 & 0.4425 & 0.5276 & 0.8130 & 1.1399 & 1.1888 \\
\hline & $k_{13}$ & 0.3463 & 0.3894 & 0.4806 & 0.7251 & 0.9704 & 1.0037 \\
\hline & $k_{14}$ & 0.3463 & 0.3894 & 0.4806 & 0.7251 & 0.9704 & 1.0037 \\
\hline
\end{tabular}

Table 3. Continued... 


\begin{tabular}{|c|c|c|c|c|c|c|c|}
\hline \multirow[t]{14}{*}{$\sigma^{2}=25$} & $k_{1}$ & 2.9064 & 3.0897 & 3.2671 & 3.7431 & 4.1254 & 4.0843 \\
\hline & $k_{2}$ & 0.9982 & 1.0378 & 1.1058 & 1.4217 & 5.6537 & 9.1050 \\
\hline & $k_{3}$ & 16.9697 & 17.2122 & 17.8079 & 20.0133 & 14.2235 & 8.6603 \\
\hline & $k_{4}$ & 1.1140 & 1.2650 & 1.4631 & 1.7424 & 1.9170 & 1.9175 \\
\hline & $k_{5}$ & 0.9257 & 0.9421 & 0.9604 & 0.9892 & 1.0029 & 1.0037 \\
\hline & $k_{6}$ & 3.1264 & 2.6384 & 2.1896 & 2.8993 & 13.1149 & 11.2494 \\
\hline & $k_{7}$ & 2.9064 & 3.0897 & 3.2671 & 3.7431 & 4.1256 & 4.1041 \\
\hline & $k_{8}$ & 2.9064 & 3.0897 & 3.2671 & 3.7431 & 4.1254 & 4.0843 \\
\hline & $k_{9}$ & 2.9105 & 3.0921 & 3.2686 & 3.7443 & 4.1266 & 4.0855 \\
\hline & $k_{10}$ & 0.9257 & 0.9421 & 0.9604 & 0.9892 & 1.0029 & 1.0037 \\
\hline & $k_{11}$ & 0.9257 & 0.9421 & 0.9604 & 0.9892 & 1.0029 & 1.0037 \\
\hline & $k_{12}$ & 1.7400 & 1.4874 & 1.2526 & 1.1957 & 1.1933 & 1.1878 \\
\hline & $k_{13}$ & 0.9257 & 0.9421 & 0.9604 & 0.9892 & 1.0029 & 1.0037 \\
\hline & $k_{14}$ & 0.9257 & 0.9421 & 0.9604 & 0.9892 & 1.0029 & 1.0037 \\
\hline \multirow[t]{14}{*}{$\sigma^{2}=100$} & $k_{1}$ & 3.5480 & 3.7782 & 3.8378 & 3.9303 & 4.1303 & 4.0535 \\
\hline & $k_{2}$ & 1.0826 & 1.1057 & 1.1632 & 1.4297 & 5.8513 & 9.6707 \\
\hline & $k_{3}$ & 49.5670 & 46.2030 & 39.5045 & 26.5260 & 14.2741 & 8.5836 \\
\hline & $k_{4}$ & 1.2200 & 1.3618 & 1.5508 & 1.7847 & 1.9492 & 1.9009 \\
\hline & $k_{5}$ & 0.9974 & 0.9988 & 1.0012 & 1.0030 & 1.0034 & 1.0039 \\
\hline & $k_{6}$ & 3.8769 & 3.0262 & 2.4780 & 3.0913 & 14.9276 & 15.7086 \\
\hline & $k_{7}$ & 3.5480 & 3.7782 & 3.8378 & 3.9304 & 4.1305 & 4.0725 \\
\hline & $k_{8}$ & 3.5480 & 3.7782 & 3.8378 & 3.9303 & 4.1303 & 4.0535 \\
\hline & $k_{9}$ & 3.5538 & 3.7816 & 3.8398 & 3.9317 & 4.1315 & 4.0546 \\
\hline & $k_{10}$ & 0.9974 & 0.9988 & 1.0012 & 1.0030 & 1.0034 & 1.0039 \\
\hline & $k_{11}$ & 0.9974 & 0.9988 & 1.0012 & 1.0030 & 1.0034 & 1.0039 \\
\hline & $k_{12}$ & 1.9897 & 1.6493 & 1.3298 & 1.2169 & 1.1932 & 1.1853 \\
\hline & $k_{13}$ & 0.9974 & 0.9988 & 1.0012 & 1.0030 & 1.0034 & 1.0039 \\
\hline & $k_{14}$ & 0.9974 & 0.9988 & 1.0012 & 1.0030 & 1.0034 & 1.0039 \\
\hline \multirow[t]{14}{*}{$\sigma^{2}=1000$} & $k_{1}$ & 3.7254 & 3.7503 & 3.7826 & 4.0203 & 4.1284 & 4.0442 \\
\hline & $k_{2}$ & 1.0901 & 1.1105 & 1.1653 & 1.4625 & 5.8678 & 16.7318 \\
\hline & $k_{3}$ & 60.1782 & 51.4491 & 40.8727 & 27.5066 & 14.2625 & 8.5588 \\
\hline & $k_{4}$ & 1.2256 & 1.3545 & 1.5600 & 1.8112 & 1.9025 & 1.9017 \\
\hline & $k_{5}$ & 1.0021 & 1.0032 & 1.0039 & 1.0040 & 1.0041 & 1.0038 \\
\hline & $k_{6}$ & 4.1366 & 3.0482 & 2.5878 & 3.4286 & 15.2173 & 18.7290 \\
\hline & $k_{7}$ & 3.7254 & 3.7503 & 3.7827 & 4.0203 & 4.1286 & 4.0638 \\
\hline & $k_{8}$ & 3.7254 & 3.7503 & 3.7826 & 4.0203 & 4.1284 & 4.0442 \\
\hline & $k_{9}$ & 3.7315 & 3.7538 & 3.7847 & 4.0217 & 4.1296 & 4.0453 \\
\hline & $k_{10}$ & 1.0021 & 1.0032 & 1.0039 & 1.0040 & 1.0041 & 1.0038 \\
\hline & $k_{11}$ & 1.0021 & 1.0032 & 1.0039 & 1.0040 & 1.0041 & 1.0038 \\
\hline & $k_{12}$ & 2.0508 & 1.6480 & 1.3267 & 1.2237 & 1.1944 & 1.1851 \\
\hline & $k_{13}$ & 1.0021 & 1.0032 & 1.0039 & 1.0040 & 1.0041 & 1.0038 \\
\hline & $k_{14}$ & 1.0021 & 1.0032 & 1.0039 & 1.0040 & 1.0041 & 1.0038 \\
\hline
\end{tabular}

Table 3. Continued... 


\begin{tabular}{|c|c|c|c|c|c|c|c|}
\hline \multirow[t]{14}{*}{$\sigma^{2}=5$} & $k_{1}$ & 0.0243 & 0.0254 & 0.0397 & 0.1045 & 0.7290 & 3.7906 \\
\hline & $k_{2}$ & 0.0239 & 0.0251 & 0.0390 & 0.0998 & 0.4969 & 5.0480 \\
\hline & $k_{3}$ & 0.0468 & 0.0497 & 0.0668 & 0.1485 & 1.0749 & 11.2802 \\
\hline & $k_{4}$ & 0.0239 & 0.0251 & 0.0391 & 0.1002 & 0.5471 & 1.7908 \\
\hline & $k_{5}$ & 0.0239 & 0.0251 & 0.0390 & 0.0998 & 0.4898 & 0.9921 \\
\hline & $k_{6}$ & 0.0247 & 0.0251 & 0.0390 & 0.0998 & 0.4913 & 1.5512 \\
\hline & $k_{7}$ & 0.0243 & 0.0254 & 0.0397 & 0.1045 & 0.7290 & 3.7906 \\
\hline & $k_{8}$ & 0.0243 & 0.0254 & 0.0397 & 0.1045 & 0.7290 & 3.7906 \\
\hline & $k_{9}$ & 0.0243 & 0.0254 & 0.0397 & 0.1045 & 0.7290 & 3.7907 \\
\hline & $k_{10}$ & 0.0239 & 0.0251 & 0.0390 & 0.0998 & 0.4898 & 0.9921 \\
\hline & $k_{11}$ & 0.0239 & 0.0251 & 0.0390 & 0.0998 & 0.4898 & 0.9921 \\
\hline & $k_{12}$ & 0.0240 & 0.0251 & 0.0391 & 0.1002 & 0.5102 & 1.1299 \\
\hline & $k_{13}$ & 0.0239 & 0.0251 & 0.0390 & 0.0998 & 0.4898 & 0.9921 \\
\hline & $k_{14}$ & 0.0239 & 0.0251 & 0.0390 & 0.0998 & 0.4898 & 0.9921 \\
\hline \multirow[t]{14}{*}{$\sigma^{2}=25$} & $k_{1}$ & 0.4410 & 0.4626 & 0.6882 & 1.4820 & 3.2961 & 3.8787 \\
\hline & $k_{2}$ & 0.3197 & 0.3394 & 0.4646 & 0.7304 & 1.0520 & 24.7335 \\
\hline & $k_{3}$ & 0.9101 & 1.0563 & 1.5380 & 3.5267 & 19.4580 & 12.3470 \\
\hline & $k_{4}$ & 0.3202 & 0.3409 & 0.4708 & 0.7824 & 1.4402 & 1.8268 \\
\hline & $k_{5}$ & 0.3194 & 0.3391 & 0.4637 & 0.7253 & 0.9632 & 1.0035 \\
\hline & $k_{6}$ & 0.3561 & 0.3541 & 0.4842 & 0.7836 & 1.2705 & 20.2522 \\
\hline & $k_{7}$ & 0.4410 & 0.4626 & 0.6882 & 1.4820 & 3.2961 & 3.8787 \\
\hline & $k_{8}$ & 0.4410 & 0.4626 & 0.6882 & 1.4820 & 3.2961 & 3.8787 \\
\hline & $k_{9}$ & 0.4410 & 0.4626 & 0.6882 & 1.4821 & 3.2962 & 3.8788 \\
\hline & $k_{10}$ & 0.3194 & 0.3391 & 0.4637 & 0.7253 & 0.9632 & 1.0035 \\
\hline & $k_{11}$ & 0.3194 & 0.3391 & 0.4637 & 0.7253 & 0.9632 & 1.0035 \\
\hline & $k_{12}$ & 0.3635 & 0.3660 & 0.4958 & 0.7920 & 1.0893 & 1.1423 \\
\hline & $k_{13}$ & 0.3194 & 0.3391 & 0.4637 & 0.7253 & 0.9632 & 1.0035 \\
\hline & $k_{14}$ & 0.3194 & 0.3391 & 0.4637 & 0.7253 & 0.9632 & 1.0035 \\
\hline \multirow[t]{14}{*}{$\sigma^{2}=100$} & $k_{1}$ & 2.4891 & 2.5443 & 2.8634 & 3.4213 & 3.8460 & 3.9557 \\
\hline & $k_{2}$ & 0.8852 & 0.8931 & 0.9384 & 0.9922 & 1.1054 & 29.2807 \\
\hline & $k_{3}$ & 14.5413 & 16.3185 & 23.8444 & 48.2369 & 60.7924 & 12.7026 \\
\hline & $k_{4}$ & 0.8875 & 0.9006 & 0.9614 & 1.0892 & 1.5196 & 1.8298 \\
\hline & $k_{5}$ & 0.8834 & 0.8909 & 0.9349 & 0.9820 & 1.0005 & 1.0037 \\
\hline & $k_{6}$ & 1.0625 & 1.0309 & 1.0719 & 1.1883 & 1.8081 & 58.6681 \\
\hline & $k_{7}$ & 2.4891 & 2.5443 & 2.8634 & 3.4213 & 3.8460 & 3.9557 \\
\hline & $k_{8}$ & 2.4891 & 2.5443 & 2.8634 & 3.4213 & 3.8460 & 3.9557 \\
\hline & $k_{9}$ & 2.4895 & 2.5445 & 2.8635 & 3.4214 & 3.8461 & 3.9558 \\
\hline & $k_{10}$ & 0.8834 & 0.8909 & 0.9349 & 0.9820 & 1.0005 & 1.0037 \\
\hline & $k_{11}$ & 0.8834 & 0.8909 & 0.9349 & 0.9820 & 1.0005 & 1.0037 \\
\hline & $k_{12}$ & 1.3734 & 1.1742 & 1.1306 & 1.1332 & 1.1399 & 1.1447 \\
\hline & $k_{13}$ & 0.8834 & 0.8909 & 0.9349 & 0.9820 & 1.0005 & 1.0037 \\
\hline & $k_{14}$ & 0.8834 & 0.8909 & 0.9349 & 0.9820 & 1.0005 & 1.0037 \\
\hline
\end{tabular}

Table 3. Continued... 


\begin{tabular}{cccccccc}
$\sigma^{2}=1000$ & $k_{1}$ & 3.5859 & 3.5177 & 3.6323 & 3.7691 & 3.8553 & 3.9229 \\
& $k_{2}$ & 1.0022 & 1.0050 & 1.0067 & 1.0143 & 1.1094 & 29.0332 \\
& $k_{3}$ & 9.2437 & 7.8368 & 4.7126 & 4.7090 & 9.8025 & 12.6319 \\
& $k_{4}$ & 1.0050 & 1.0143 & 1.0330 & 1.1165 & 1.5192 & 1.8106 \\
& $k_{5}$ & 0.9999 & 1.0023 & 1.0027 & 1.0035 & 1.0040 & 1.0038 \\
& $k_{6}$ & 1.2731 & 1.1681 & 1.1801 & 1.2222 & 1.9098 & 63.1879 \\
& $k_{7}$ & 3.5859 & 3.5177 & 3.6323 & 3.7691 & 3.8553 & 3.9229 \\
& $k_{8}$ & 3.5859 & 3.5177 & 3.6323 & 3.7691 & 3.8553 & 3.9229 \\
$k_{9}$ & 3.5866 & 3.5181 & 3.6325 & 3.7692 & 3.8555 & 3.9230 \\
$k_{10}$ & 0.9999 & 1.0023 & 1.0027 & 1.0035 & 1.0040 & 1.0038 \\
$k_{11}$ & 0.9999 & 1.0023 & 1.0027 & 1.0035 & 1.0040 & 1.0038 \\
$k_{12}$ & 1.7056 & 1.3760 & 1.2385 & 1.1651 & 1.1437 & 1.1427 \\
$k_{13}$ & 0.9999 & 1.0023 & 1.0027 & 1.0035 & 1.0040 & 1.0038 \\
$k_{14}$ & 0.9999 & 1.0023 & 1.0027 & 1.0035 & 1.0040 & 1.0038 \\
\hline
\end{tabular}

\section{Discussion}

From Table 2, it is observed that for a moderate to high degree of correlations, the suggested estimators perform better than all the other existing estimators and coincide with $k_{5}$. From Table 3, one may observe that the estimators $k_{2}, k_{3}$ and $k_{6}$ i.e., due to Lawless-Wang [10], Nomura [19] and Dorugade [16], have shown somewhat peculiar behaviour than any other estimators. Further for large $n$, say $n \geq 50$, low error variance $\sigma^{2}$ $(\leq 5)$, low and moderate degree of correlations $(\rho)$, all the estimators considered here have produced unstable estimates for the ridge parameter. Estimators due to $k_{1}$ i.e., Hoerl et al. [18], Satish and Vidya [20,21,24] i.e., $k_{7}, k_{8}$ and $k_{9}$ behaved better, and yielded more stable estimates to the regression coefficients, but it is observed carefully that, these estimators slightly over shrinks the estimates to the regression coefficients as compared to other estimators due to Dorugade and Kashid [15], Satish and Vidya [24] i.e., $k_{10}, k_{11}$, and, $k_{12}$; and the proposed estimators $k_{13}$ and $k_{14}$.

\section{Conclusion}

Motivated by the interesting behaviour of several popular estimators in Ridge Regression, an attempt was made to further refine the estimators so that the Mean Square Errors are reduced to a certain extent possible so that more robust and reliable estimates can be achieved. Accordingly new estimators were developed and their performance was evaluated in this study. As standard procedures to establish the robustness of the estimators, the newly developed estimators were evaluated in terms of MSE and compared with existing methods of estimators. Examples using real-life data and simulation studies were provided to illustrate the practical benefits of the new estimators. In all cases the performance of the proposed estimators was satisfactory and comparable. 


\section{Acknowledgment}

First author is immensely grateful to Professor C. Raju., Professor in quantitative Methods and Operations management, Indian institute of Management, Kozhikode, Kerala, India, for his invaluable suggestions in writing this article.

\section{References}

1. D. W. Marquardt and R. D. Snee, American Statistician 29, 3 (1975). https://doi.org/10.1080/00031305.1975.10479105

2. H. D. Vinod and A. Ullah, Recent Advances in Regression Methods (Marcel Dekker, New York, 1981).

3. A. E. Hoerl, Chem. Eng. Prog. 58, 54 (1962). https://doi.org/10.1021/ie50632a904

4. A. E. Hoerl and R. W. Kennard, Technometrics 12, 69 (1970). https://doi.org/10.1080/00401706.1970.10488635

5. I. S. Helland, Scanndavion J. Stat. 17, 97 (1990). https://doi.org/10.1111/j.1365-2559.1990.tb00673.x

6. S. Mardikyan and E. Cetin, Int. J. Contemp. Math. Sci. 3, 527, (2008).

7. R. Tibshirani, J. Royal Stat. Soc. Series B (methodological) 58, 267 ((1996). https://doi.org/10.1111/j.2517-6161.1996.tb02080.x

8. A. M. Halawa and M. Y. El Bassiouni, J. Stat. Comput. Simul. 65, 341 (2000). https://doi.org/10.1080/00949650008812006

9. T. Hastie and R. Tibshirani, Generalised Additive Models (Chapman \& Hall/CRC, London, 1990).

10. J. F. Lawless and P. Wang, Commun. Statist. Theory Methods 5, 307 (1976). https://doi.org/10.1080/03610927608827361

11. B. M. Kibria Commun. Statist-Simulation Comput. 32, 419 (2003). https://doi.org/10.1081/SAC-120017499

12. G. Khalaf and G. Shukur, Commun. Statist. Theory Methods 34, 1177 (2005). https://doi.org/10.1081/STA-200056836

13. G. Khalaf, J. Mod. Appl. Stat. Method 11, 443 (2012). https://doi.org/10.22237/jmasm/1351743240

14. G. Muniz and B. M. Kibria, Commun. Statistics-Simul. Comput. 38, 621 (2009). https://doi.org/10.1080/03610910802592838

15. A. V. Dorugade and D. N. Khashid, Appl. Math. Sci. 4, 447 (2010).

16. A. V. Dorugade, J. Assoc. Arab Univ. Basic Appl. Sci. 15, 94 (2014). https://doi.org/10.1016/j.jaubas.2013.03.005

17. Y. M. Al-Hassan, J. Assoc. Arab Univ. Basic Appl. Sci. 9, 23 (2010). https://doi.org/10.1016/j.jaubas.2010.12.006

18. A. E. Hoerl, R. W. Kennard, and K. F. Baldwin, Commun. Statist. Theory Methods 4, 105 (1975). https://doi.org/10.1080/03610917508548342

19. M. Nomura, Commun. Statistics - Simul. Comput. 17, 729 (1988). https://doi.org/10.1080/03610918808812690

20. S. Bhat and R. Vidya, Improvement on Ridge Regression Estimator - Proc. of National Conference on 'Statistical Methods and Data Analysis (2015) pp. 65-71.

21. S. Bhat and R. Vidya, Pak. J. Stat. Operations Res. (PJSOR), 12, 317 (2016). https://doi.org/10.18187/pjsor.v12i2.1188

22. D. C. Montgomery, E. A. Peck and G. G. Vining, Introduction to Linear Regression Analysis, $3^{\text {rd }}$ Edition (John Wiley and Sons, Singapore, 2003).

23. K. Liu, Commun. Statist. Theory Methods 32, 1009 (2003). https://doi.org/10.1081/STA$\underline{120019959}$ 
24. S. Bhat and R. Vidya, A New Ridge Estimator and Its Performance - Proc. of the National Conference on Operations Management, Analytics and Statistical Methods (2017).

25. S. Bhat, Int. J. Agri. Stat. Sci. 15, 347 (2019).

26. R. G. Askin and D. C. Montgomery, Int. J. Sci.: Basic Appl. Res. (IJSBAR), 22, 333 (1980). https://doi.org/10.2307/1268317

27. P. W. Holland, NBER Working Paper Series 11, 1 (1973).

28. E. R. Berndt, The Practice of Econometrics (Addison -Wesley, NY, 1991).

http://lib.stat.cmu.edu/datasets/CPS_85_Wages. 